\title{
On Human Capital and Team Stability ${ }^{1}$
}

\author{
Pierre-André Chiappori ${ }^{2}$ \\ Alfred Galichon ${ }^{3}$ \\ Columbia University \\ NYU and Sciences Po \\ Bernard Salanié ${ }^{4}$ \\ Columbia University
}

August 13, 2017

\begin{abstract}
${ }^{1}$ We thank Yeon-Koo Che, Navin Kartik, the editor and referees, and especially Arnaud Dupuy, Fuhito Kojima and Phil Reny for useful comments. This paper builds on material from an unpublished manuscript circulated under the name "The Roommate Problem Is More Stable Than You Think," which is now obsolete.

Accepted for publication by the Journal of Human Capital, Volume 13, Number 2, Summer 2019. URL: https://doi.org/10.1086/702925.

${ }^{2}$ Address: Department of Economics, Columbia University, 1009A International Affairs Building, 420 West 118th St., New York, NY 10027, USA. E-mail: pc2167@columbia.edu. Chiappori gratefully acknowledges financial support from the NSF (award \#1124277), and ANR (grant Famineq).

${ }^{3}$ Economics Department and Courant Institute, New York University and Economics Department, Sciences Po. Address: NYU, Department of Economics. 19 W 4th Street, New York, NY 10012, USA. Email: ag133@nyu.edu. Galichon gratefully acknowledges funding from NSF grant DMS-1716489, ERC grants FP7-295298, FP7-312503, FP7-337665, and ANR grant Famineq.

${ }^{4}$ Address: Department of Economics, Columbia University, 1131 International Affairs Building, 420 West 118th Street, New York, NY 10027, USA. E-mail: bsalanie@columbia.edu.
\end{abstract}




\begin{abstract}
In many economic contexts, agents from a same population team up to better exploit their human capital. In such contexts (often called "roommate matching problems"), stable matchings may fail to exist even when utility is transferable. We show that when each individual has a close substitute, a stable matching can be implemented with minimal policy intervention. Our results shed light on the stability of partnerships on the labor market. Moreover, they imply that the tools crafted in empirical studies of the marriage problem can easily be adapted to many roommate problems.
\end{abstract}




\section{Introduction}

Among Gary Becker's seminal contributions to labor economics, two are of particular importance. The most obvious one is the notion of human capital, after which this Journal is named. A second important contribution is the development of matching models with transferable utility ${ }^{1}$. Although the market for marriage was Becker's favorite field of application for the theory, many of the insights he developed are deeply relevant for the analysis of labor issues as well. The labor market can often be fruitfully seen as matching people to jobs - an insight that has been thoroughly exploited in the literature, in particular in a search context ${ }^{2}$.

There is, however, a fundamental difference between matching on the labor and the marriage market. In the latter case, bilateral matching is a natural framework; individuals who match mostly belong to two distinct subpopulations. Not so, however, on the labor market. Workers match not only to jobs, but also (and often primarily) to other workers. Lawyers gather in law firms, doctors associate in medical practices, architects congregate in architectural firms. While such partnerships are typical of the professions, they extend to other services firms such as consultancies. More generally, the notion that workers, through their employment relationships, tend to match to other workers with similar characteristics, has received a lot of attention and clear empirical support. For instance, a recent paper by Ehrlich and Kim, 2015 shows that immigrants endowed with similar skills tend to team up and/or to separate in the same sectors. In particular, higher levels of human capital of specific skill groups in the destination country tend to increase the immigration flows of corresponding groups from the source country. The authors convincingly argue that these effects are crucial in assessing the economic consequences of migrations.

From a theoretical perspective, these features raise specific problems. In sharp contrast with the bipartite literature, the formal analysis of workers' matching on human capital must acknowledge the fact that the individuals under consideration typically belong to the same population. It has been known for some time that this apparently minor difference in settings may generate largely divergent properties. Take, for instance, the specific case in which teams consist of exactly two people, both coming from the same population: this is classically called the roommate matching problem.

The standard equilibrium concept in matching is stability; a matching

\footnotetext{
${ }^{1}$ See for instance Becker, 1973 and Becker, 1974.

${ }^{2}$ See in particular Mortensen and Pissarides, 1994.
} 
is stable if it is robust to unilateral and bilateral deviations. We will follow this long tradition in this paper: when we say that "an equilibrium exists", for instance, we mean that "a stable matching exists". Can we expect that the roommate matching game always has a stable matching, so that the theoretical analysis could, as in the bipartite framework, concentrate on the properties and the comparative statics of this stable outcome? Or could it be the case that a stable matching fails to exist, which might cast serious doubts on the relevance of matching models for the analysis of these situations?

The answer to that important question has been known for a long time in the Non Transferable Utility (NTU) context; indeed, Gale and Shapley, 1962 have shown that stable matchings may not exist. However, applying the NTU approach to a labor market requires wages to be exogenously fixed ,rather than being endogenously determined at equilibrium. In most markets, this is not the relevant framework. Much more adequate is a Transferable Utility (TU) framework, in which any potential team generates a surplus that is (endogenously) shared by its members.

The problem, however, is that roommates matching games under TU tend to have different properties than their bipartite counterparts. In a bipartite setting, a stable matching exists under mild continuity and compactness conditions; it maximizes aggregate surplus, and the associated individual surpluses solve the dual imputation problem. A first conclusion of the present paper is that in the two-partner roommate matching problem under TU, on the contrary, stable matching may fail to exist. This is a potentially damaging conclusion, since it might require reconsidering the relevance of matching models in this context.

Our second conclusion, however, tends to mitigate this negative result by showing that its economic implications may be much less damaging than one would expect. Specifically, we consider a model in which agents belong to various "types", where each type consists of individuals of indistinguishable characteristics and tastes. In this context, we show two main results. First, a stable matching always exists when the number of individuals in each type is even. Second, when the number of individuals of any given type is large enough, there always exist "quasi-stable" matchings: even if a stable matching does not exist, existence can be restored with minimal policy intervention. To do this, one only needs to convince one individual to leave the game in each type with an odd number of individuals. If this requires a compensation to be paid, this can be done at a per capita cost that goes to zero when the population of each type goes to infinity.

We refer the reader to our conclusion for the implications of these findings in terms of the stability of partnerships. We also show there that the 
empirical tools devised for the bipartite matching setting ${ }^{3}$ should carry over directly to the roommate context when the populations under consideration are large. Some of the results of the present paper are applied in this direction in Ciscato et al., 2015.

Existing literature Since Gale and Shapley, 1962, a few papers have studied the property of NTU stable roommate matchings when they do exist. Gusfield and Irving, 1989 showed that the set of singles is the same in all stable matchings; Klaus and Klijn, 2010 study whether any of them can be "fair". Efficient algorithms have also been available since Irving, 1985. Necessary and sufficient existence conditions under strict preferences have been found by Tan, 1991 for complete stable matchings and by Sotomayor, 2005 for stable matchings. Chung, 2000 shows that a condition he calls "no odd rings" is sufficient for stable matchings to exist under weak preferences. Rodrigues-Neto, 2007 introduces "symmetric utilities" and Gudmunsson, 2014 uses "weak cycles."

The TU case has been less studied in the theoretical literature, in spite of its relevance in empirical applications. Chung, 2000 shows that when the division of surplus obeys an exogenous rule, odd rings are ruled out and the roommate problem has a stable matching; but that is clearly not an appealing assumption. Karlander and Eriksson, 2001 provide a graphtheoretic characterization of stable outcomes when they exist; and Klaus and Nichifor, 2010 studies their properties. Talman and Yang, 2011 give a characterization in terms of integer programming.

The results of this paper are also related to those of Azevedo et al., 2013, who show the existence of a Walrasian equilibrium in an economy with indivisible goods, a continuum of agents and quasilinear utility. Unlike their main results, ours apply in markets with finite numbers of agents. Our methods are also original. As is well-known, in bipartite problems all feasible matchings that maximize social surplus are stable. This is not true in roommate problems; but we show how any roommate problem can be "cloned" in order to construct an associated bipartite problem. We then exploit this insight to prove existence of stable matchings in roommate problems with even numbers of agents within each type.

To the best of our knowledge, the connection between the unipartite and bipartite problems stressed in this paper is new.

\footnotetext{
${ }^{3}$ See Chiappori and Salanié, 2016 for a recent survey.
} 


\section{A Simple Example}

We start by giving the intuition of our main results on an illustrative example.

\subsection{Unstable Matchings}

It has been known since Gale and Shapley that a stable matching may not exist for the roommate problem under non-transferable utility. As it turns out, it is almost equally easy to construct an example of non-existence of a stable matching with transferable utility. Here a matching defines who is matched to whom and how the corresponding surplus is divided between the partners. Stability requires that

- no partner would be better off by leaving the partnership

- no group of individuals could break off their current match, rematch together, and generate a higher joint surplus than the sum of their current individual utilities.

Consider the following example, in which only two-member matches are possible:

Example 1 The population has three individuals. Any unmatched individual has zero utility. The joint surplus created by the matching of any two of them is given by the off-diagonal terms of the matrix

$$
\Phi=\left(\begin{array}{ccc}
- & 6 & 8 \\
6 & - & 5 \\
8 & 5 & -
\end{array}\right)
$$

so that individuals 1 and 2 create, if they match, a surplus of $6 ; 1$ and 3 create a surplus of 8 , etc.

Assume that there exists a stable matching. A matching in which all individuals remain single is obviously not stable; any stable matching must be such that one person remains single and the other two are matched together. Let $\left(u_{x}\right)$ be the utility that individual of type $x=1,2,3$ gets out of this game; stability imposes $u_{x}+u_{y} \geq \Phi_{x y}$ for all potential matches, with equality if $x$ and $y$ are actually matched - and $u_{x} \geq 0$ with equality if $x$ is single. One can readily check, however, that no set of numbers $\left(u_{1}, u_{2}, u_{3}\right)$ satisfying these relationships for all $x$ and $y$ exists: whichever the married pair is, one of 
the matched partners would increase her utility by matching with the single person. Indeed, if the matched pair is $\{1,2\}$, then

$$
u_{1}+u_{2}=6, u_{3}=0, u_{2} \geq 0
$$

contradicts $u_{1}+u_{3} \geq 8$ : agent 3, being single, is willing to give up any amount smaller than 8 to be matched with 1, while the match between 1 and 2 cannot provide 1 with more than 6 . Similarly, if the married pair is $\{2,3\}$, then

$$
u_{2}+u_{3}=5, u_{1}=0, u_{2} \geq 0, u_{3} \geq 0
$$

contradicts both $u_{1}+u_{2} \geq 6$ and $u_{1}+u_{3} \geq 8$ (so that 1 is willing to give more than 5 and less than 6 to agent 2 to match with her, and more than 5 and less than 8 to 3.) Finally, if the married pair is $\{1,3\}$, then

$$
u_{1}+u_{3}=8, u_{2}=0, u_{1} \geq 0, u_{3} \geq 0
$$

is incompatible with $u_{1}+u_{3} \geq 11$, which follows from combining $u_{1}+u_{2} \geq 6$ and $u_{2}+u_{3} \geq 5$ with $u_{2}=0$ (since agent 2 is single 1 could match with her and capture almost 6, while 3 could match with her and capture almost 5; these outside options are more attractive than anything 1 and 3 can achieve together.) We conclude that no stable matching exists.

Note that there is nothing pathological in Example 1. The surpluses can easily be (locally) modified without changing the result. Also, the conclusion does not require an odd number of agents; one can readily introduce a fourth individual, who generates a small enough surplus with any roommate, without changing the non-existence finding.

\subsection{Cloning}

However, there exists a simple modification that restores existence in Example 1. Let us now duplicate the economy by "cloning" each agent; technically, we now have three types $x=1,2,3$ of agents, with two (identical) individuals of each type. The joint surplus created by a matching between two individuals of different types $x \neq y$ is as in Example 1; but we now also need to define the surplus generated by the matching of two clones (two individuals of the same type.) Take it to be 2 for every type -more on this later. We then have the matrix:

$$
\Phi^{\prime}=\left(\begin{array}{lll}
2 & 6 & 8 \\
6 & 2 & 5 \\
8 & 5 & 2
\end{array}\right)
$$


Consider the following matching $\mu^{*}$ : there is one match between a type 1 and a type 2 individuals, one between type 1 and type 3 , and one between type 2 and type 3 . Assume individuals share the surplus so that each individual of type 1 gets 4.5 , each individual of type 2 gets 1.5, and each individual of type 3 gets 3.5 . This is clearly feasible; and it is easy to verify that it is a stable matching.

Less obvious but still true is the fact (proved later on) that existence would still obtain for any values chosen for the diagonal of the matrix, although the stable matching pattern that would emerge may be different ${ }^{4}$. In other words, our cloning operation always restores the existence of a stable match, irrespective of the values of the joint surpluses created by matches between clones.

\subsection{Surplus Maximization}

Our main result is better understood when related to another, closely linked problem: finding a feasible matching that maximizes total surplus. Total surplus is simply the sum of the joint surpluses of every match (keeping to a normalized utility of zero for singles). In the standard, bipartite framework, the adjective "feasible" refers to the fact that each individual can only be matched to one partner or stay single. Roommate matching, however, introduces an additional feasibility constraint. For any two types $x \neq y$, denote $\mu_{x y}$ the number of matches between an individual of type $x$ and an individual of type $y$; since a roommate matching for which $\mu_{x y}$ and $\mu_{y x}$ differ would clearly not be feasible, it must be the case that $\mu_{x y}=\mu_{y x}$. This additional symmetry constraint is absent from the bipartite model, where these two individuals would belong to two separate subpopulations and the number of marriages between say, a college-educated man and a woman who is a high-school graduate may well differ (and typically does) from the number of marriages between a college-educated woman and a man who is a high-school graduate.

This symmetry constraint is the source of the difficulty in finding stable roommate matchings; and our cloning operation addresses it. To see this on our Example 1, first go back to roommate matching with one individual of each type $x=1,2,3$, and neglect the symmetry constraint. Since there is only one individual of each type $x$, she cannot match with herself: $\mu_{x x} \equiv 0$;

\footnotetext{
${ }^{4}$ For instance, if the diagonal elements are large enough, the stable matching matches each individual with her clone.
} 
and neglecting symmetry, the only other feasibility constraints are

$$
\text { for every } x, \sum_{y \neq x} \mu_{x y} \leq 1
$$

and

$$
\text { for every } y, \sum_{x \neq y} \mu_{x y} \leq 1
$$

The two matchings

$$
\mu^{1}=\left(\begin{array}{lll}
0 & 0 & 1 \\
1 & 0 & 0 \\
0 & 1 & 0
\end{array}\right) \text { and } \mu^{2}=\left(\begin{array}{lll}
0 & 1 & 0 \\
0 & 0 & 1 \\
1 & 0 & 0
\end{array}\right)
$$

are feasible in this limited sense; and they both achieve the highest possible surplus when the symmetry conditions are disregarded. The existence of two solutions is not surprising: given the symmetric nature of the surplus matrix $\Phi$, if a matrix $\mu$ maximizes total surplus, so does its transpose $\mu^{t}$. Unfortunately, neither is symmetric, and therefore neither makes any sense in the roommate problem. For instance, $\mu^{1}$ has agent 1 matched both with agent 3 (in the first row) and with agent 2 (in the first column). Also, note that a third solution to this relaxed problem is the unweighted mean of $\mu^{1}$ and $\mu^{2}$,

$$
\mu^{m}=\left(\begin{array}{ccc}
0 & 1 / 2 & 1 / 2 \\
1 / 2 & 0 & 1 / 2 \\
1 / 2 & 1 / 2 & 0
\end{array}\right)
$$

However, while this matrix is indeed symmetric, its coefficients are not integer and thus it is not a feasible matching either; moreover, and quite interestingly, it cannot be interpreted as the outcome of randomization since it is not a convex combination of feasible roommate matching matrices ${ }^{5}$.

Let us now reintroduce the symmetry constraint. The (now fully) feasible matching that maximizes total surplus can only have one matched pair and one single; and the pair that should be matched clearly consists of individuals 1 and 3:

$$
\bar{\mu}=\left(\begin{array}{lll}
0 & 0 & 1 \\
0 & 0 & 0 \\
1 & 0 & 0
\end{array}\right)
$$

\footnotetext{
${ }^{5}$ For any stable roommate matching matrix, the sum of coefficients equals 2, reflecting the fact that one agent must remain single. This property is preserved by convex combination; however, the sum of coefficients of $\mu^{m}$ equals 3.
} 
Obviously, $\bar{\mu}$ is not a solution to the maximization problem without symmetry constraint; in other words, the symmetry constraint is binding in this example. As we shall see below, this is characteristic of situations in which the roommate matching problem with transferable utility does not have a stable matching. Indeed, we prove in the next section that a stable matching exists if and only if the symmetry constraint does not bind.

Now take the "cloned" version of Example 1, in which each type $x$ has two individuals. It is easy to see that the solution to the relaxed problem which neglects the symmetry constraint is the $\mu^{*}$ of section 2.2 , which is symmetric; therefore the symmetry constraint does not bind, and a stable matching exists. This is a general result: we shall see below that in any cloned roommate matching setup, at least one solution to the relaxed problem is symmetric - which implies the existence of a stable match.

\subsection{A Bipartite Interpretation}

The relaxed problem, in turn, has a natural interpretation in terms of bipartite matching. Start from the three-agent Example 1, and define an associated bipartite matching problem as follows: clone the population again, but this time assign a label (such as "man" or "woman") to each of the two subpopulations. Then consider the bipartite matching problem between these subpopulations of "men" and "women", with the joint surplus matrix given by $\Phi^{\prime}$ in $(2)$.

By standard results, there always exists a stable matching in this associated bipartite matching problem; and it maximizes the associated total surplus. In our example, $\mu^{1}$ and $\mu^{2}$ are the two stable matchings. Any convex combination such as $\mu^{m}$ can be interpreted as a randomization between these two matchings; it is natural to focus on $\mu^{m}$ since it is the only symmetric one and feasible roommate matchings must be symmetric. As remarked above, in the original roommate problem $\mu^{m}$ cannot be stable, since it has non-integer element.

Now if the roommate matching problem is cloned we can proceed as in the above paragraph, except that with twice the number of individuals we should work with $2 \mu^{m}$. As an integer symmetric matrix, reinterpreted in the cloned roommate matching setup, it defines a feasible roommate matching which is stable - in fact it is the stable matching $\mu^{*}$ of section 2.2. This construction is general: we shall see below that any roommate matching problem in which the number of individuals in each type is even has a symmetric stable match.

We now provide a formal derivation of these results. 


\section{The Formal Setting}

We consider a population of individuals who belong to a finite set of types $\mathcal{X}$. Individuals of the same type are indistinguishable. We denote $n_{x}$ the number of individuals of type $x \in \mathcal{X}$, and

$$
N=\sum_{x \in \mathcal{X}} n_{x}
$$

the total size of the population.

Without loss of generality, we normalize the utilities of singles to be zero throughout.

\subsection{Roommate Matching}

A match consists of two partners of types $x$ and $y$. An individual of any type can be matched with any individual of the same or any other type, or remain single. In particular, there is no restriction that matches only involve two partners of different "genders."

Let a match $\{x, y\}$ generate a surplus $\Phi_{x y}$. In principle the two partners could play different roles. In sections 3 and 4 we will assume that they are in fact symmetric within a match, so that $\Phi_{x y}$ is assumed to be a symmetric function of $(x, y)$ :

Assumption 1 The surplus $\Phi_{x y}$ is symmetric in $(x, y)$.

We show in section 5 that, surprising as it may seem, there is in fact no loss of generality in making this assumption. The intuition is simple: if $\Phi_{x y}$ fails to be symmetric in $(x, y)$, so that the partners' roles are not exchangeable, then they should choose their roles so to maximize output. This boils down to replacing $\Phi_{x y}$ with the symmetric $\max \left(\Phi_{x y}, \Phi_{y x}\right)$. Thus our results extend easily when we do not impose Assumption 1; but it is easier to start from the symmetric case.

A matching can be described by a matrix of numbers $\left(\mu_{x y}\right)$ indexed by $x, y \in \mathcal{X}$, such that

- $\mu_{x 0}$ is the number of singles of type $x$

- when $y \neq 0, \mu_{x y}$ is the number of matches between types $x$ and $y$. 
The numbers $\mu_{x y}$ should be integers; given Assumption 1, they should be symmetric in $(x, y)$; and they should satisfy the scarcity constraints. More precisely, the number of individuals of type $x$ must equal the number $\mu_{x 0}$ of singles of type $x$, plus the number of pairs in which only one partner has type $x$, plus twice the number of pairs in which the two partners are of type $x$ - since such a same-type pair has two individuals of type $x$.

Finally, the set of feasible roommate matchings is

$$
\mathcal{P}(n)=\left\{\mu=\left(\mu_{x y}\right):\left(\begin{array}{c}
2 \mu_{x x}+\sum_{y \neq x} \mu_{x y} \leq n_{x} \\
\mu_{x y}=\mu_{y x} \\
\mu_{x y} \in \mathbb{N}
\end{array}\right)\right\}
$$

\subsection{TU stability and optimality}

We define an outcome $(\mu, u)$ as the specification of a feasible roommate matching $\mu$ and an associated vector of payoffs $u_{x}$ to each individual of type $x$. These payoffs have to be feasible: that is, the sum of payoffs across the population has to be equal to the total output under the matching $\mu$. Now in a roommate matching $\mu$, the total surplus created is ${ }^{6}$

$$
S_{R}(\mu ; \Phi)=\sum_{x} \mu_{x x} \Phi_{x x}+\sum_{x \neq y} \mu_{x y} \frac{\Phi_{x y}}{2} .
$$

This leads to the following definition of a feasible outcome: an outcome $(\mu, u)$ is feasible if $\mu$ is a feasible roommate matching and

$$
\sum_{x \in \mathcal{X}} n_{x} u_{x}=S_{R}(\mu ; \Phi)
$$

We define stability as in Gale and Shapley, 1962: an outcome $(\mu, u)$ is stable if it cannot be blocked by an individual or by a pair of individuals. More precisely, an outcome $(\mu, u)$ is stable if it is feasible, and if for any two types $x, y \in \mathcal{X}$, (i) $u_{x} \geq 0$, and (ii) $u_{x}+u_{y} \geq \Phi_{x y}$. By extension, a matching $\mu$ is called stable if there exists a payoff vector $\left(u_{x}\right)$ such that the outcome $(\mu, u)$ is stable.

In bipartite matching the problem of stability is equivalent to the problem of optimality: stable matchings maximize total surplus. Things are obviously more complicated in roommate matchings - there always exist surplusmaximizing matchings, but they may not be stable. The maximum of the

\footnotetext{
${ }^{6}$ Note that in the second sum operator the pair $\{x, y\}$ appears twice, one time as $(x, y)$ and another time as $(y, x)$; but the joint surplus $\Phi_{x y}$ it creates must only be counted once, hence the division by 2 .
} 
aggregate surplus over the set of feasible roommate matchings $\mathcal{P}(n)$ is

$$
\begin{aligned}
\mathcal{W}_{\mathcal{P}}(n, \Phi)= & \max S_{R}(\mu ; \Phi) \\
\text { s.t. } & 2 \mu_{x x}+\sum_{y \neq x} \mu_{x y} \leq n_{x} \\
& \mu_{x y}=\mu_{y x} \\
& \mu_{x y} \in \mathbb{N} .
\end{aligned}
$$

While no stable matching may actually achieve this value, it plays an important role in our argument.

\subsection{The Associated Bipartite Matching Problem}

We shall now see that to every roommate matching problem we can associate a bipartite matching problem which generates almost the same level of aggregate surplus. More precisely, we will prove that for every vector of populations of types $n=\left(n_{x}\right)$ and every symmetric surplus function $\Phi=\left(\Phi_{x y}\right)$, the highest possible surplus in the roommate matching problem is "close to" that achieved in a bipartite problem with mirror populations of men and women and half the surplus function:

$$
\mathcal{W}_{\mathcal{P}}(n, \Phi) \simeq \mathcal{W}_{\mathcal{B}}(n, n, \Phi / 2) .
$$

where $\mathcal{W}_{\mathcal{B}}(n, n, \Phi / 2)$ is defined as the maximal surplus of the bipartite matching problem:

$$
\mathcal{W}_{\mathcal{B}}(n, n, \Phi / 2)=\max _{\nu \in \mathcal{B}(n, n)} S_{B}(\nu ; \Phi)
$$

where $S_{B}(\nu ; \Phi)=\sum_{x, y \in \mathcal{X}} \nu_{x y} \frac{\Phi_{x y}}{2}$ and $\mathcal{B}(n, n)$ is the set of feasible matchings in the bipartite problem:

$$
\mathcal{B}(n, n)=\left\{\nu=\left(\nu_{x y}\right):\left(\begin{array}{c}
\sum_{y} \nu_{x y} \leq n_{x} \\
\sum_{x} \nu_{x y} \leq n_{y} \\
\nu_{x y} \in \mathbb{N}
\end{array}\right)\right\}
$$

We also define stability for a feasible bipartite matching $\left(\nu_{x y}\right)$ in the usual way: there must exist payoffs $\left(u_{x}, v_{y}\right)$ such that

$$
\begin{aligned}
S_{B}(\nu ; \Phi) & =\sum_{x \in \mathcal{X}} n_{x} u_{x}+\sum_{y \in \mathcal{X}} n_{y} v_{y} \\
u_{x}+v_{y} & \geq \frac{\Phi_{x y}}{2} \\
u_{x} \geq 0, & \quad v_{y} \geq 0
\end{aligned}
$$


By classical results of Shapley and Shubik, 1971, there exist stable matchings $\nu$, and they coincide with the solutions of (7). Moreover, the associated payoffs $(u, v)$ solve the dual program; that is, they minimize $\sum_{x \in \mathcal{X}} n_{x} u_{x}+$ $\sum_{y \in \mathcal{X}} n_{y} v_{y}$ over the feasible set of program (9). Finally, for any stable matching, $\mu_{x y}>0$ implies $u_{x}+v_{y}=\Phi_{x y} / 2$, and $\mu_{x 0}>0$ implies $u_{x}=0$.

Remark 3.1 The marriage problem obviously is a particular case of the roommate problem: if in a roommate matching problem $\Phi_{x y}=-\infty$ whenever $x$ and $y$ have the same gender, then any optimal or stable matching will be heterosexual.

\subsubsection{Links Between $\mathcal{W}_{\mathcal{P}}$ and $\mathcal{W}_{\mathcal{B}}$}

It is not hard to see that $\mathcal{W}_{\mathcal{P}}(n, \Phi) \leq \mathcal{W}_{\mathcal{B}}(n, n, \Phi / 2)$. In fact, we can bound the difference between these two values:

Theorem 1 Under Assumption 1,

$$
\mathcal{W}_{\mathcal{P}}(n, \Phi) \leq \mathcal{W}_{\mathcal{B}}(n, n, \Phi / 2) \leq \mathcal{W}_{\mathcal{P}}(n, \Phi)+|\mathcal{X}|^{2} \bar{\Phi}
$$

where

$$
\bar{\Phi}=\sup _{x, y \in \mathcal{X}} \Phi_{x y} .
$$

and $|\mathcal{X}|$ is the cardinal of the set $\mathcal{X}$, i.e. the number of types in the population.

Proof. See appendix.

In some cases, $\mathcal{W}_{\mathcal{P}}(n, \Phi)$ and $\mathcal{W}_{\mathcal{B}}(n, n, \Phi / 2)$ actually coincide. For instance:

Proposition 2 If $n_{x}$ is even for each $x \in \mathcal{X}$, then under Assumption 1,

$$
\mathcal{W}_{\mathcal{P}}(n, \Phi)=\mathcal{W}_{\mathcal{B}}(n, n, \Phi / 2) \text {. }
$$

Proof. See appendix.

\subsubsection{Stable Roommate Matchings}

The existence of stable roommate matchings is directly related to the divergence of $\mathcal{W}_{\mathcal{P}}(n, \Phi)$ and $\mathcal{W}_{\mathcal{B}}(n, n, \Phi / 2)$. Indeed, one has: 
Theorem 3 Under Assumption 1,

(i) There exist stable roommate matchings if and only if

$$
\mathcal{W}_{\mathcal{P}}(n, \Phi)=\mathcal{W}_{\mathcal{B}}(n, n, \Phi / 2)
$$

(ii) Whenever they exist, stable roommate matchings achieve the maximal aggregate surplus $\mathcal{W}_{\mathcal{P}}(n, \Phi)$ in (6).

(iii) Whenever a stable roommate matching exists, individual utilities at equilibrium $\left(u_{x}\right)$ solve the following, dual program:

$$
\begin{array}{ll}
\min _{u, A} & \sum_{x} u_{x} n_{x} \\
\text { s.t. } & u_{x} \geq 0 \\
& u_{x}+u_{y} \geq \Phi_{x y}+A_{x y} \\
& A_{x y}=-A_{y x}
\end{array}
$$

Proof. See appendix.

Note that while the characterization of the existence of a stable matching in terms of equality between an integer program and a linear program is a well-known problem in the literature on matching (see Talman and Yang, 2011 for the roommate problem), the link with a bipartite matching problem is new.

Also note that in program (10), the antisymmetric matrix $A$ has a natural interpretation: $A_{x y}$ is the Lagrange multiplier of the symmetry constraints $\mu_{x y}=\mu_{y x}$ in the initial program (6). Our proof shows that if $\mu_{x y}>0$ in a stable roommate matching, then the corresponding $A_{x y}$ must be nonpositive; but since $\mu_{y x}=\mu_{x y}$ the multiplier $A_{y x}$ must also be non-positive, so that both must be zero. The lack of existence of a stable roommate matching is therefore intimately linked to a binding symmetry constraint.

Given Proposition 2, Theorem 3 has an immediate corollary: with an even number of individuals per type, there must exist a stable roommate matching. Formally:

Corollary 3.1 If $n_{x}$ is even for each $x \in \mathcal{X}$, then under Assumption 1, there exists a stable roommate matching.

In particular, for any roommate matching problem, its "cloned" version, in which each agent has been replaced with a couple of clones, has a stable matching; and this holds irrespective of the surplus generated by the 
matching of two identical individuals. Of course, in general much less than full cloning is needed to restore existence; we give this statement a precise meaning in the next paragraph.

Our next result shows that one can restore the existence of a stable matching by removing at most one individual of each type from the population; if these individuals have to be compensated for leaving the game, this can be done at limited total cost:

Theorem 4 (Approximate stability) Under Assumption 1, in a population of $N$ individuals, there exists a subpopulation of at least $N-|\mathcal{X}|$ individuals among which there exist a stable matching, where $|\mathcal{X}|$ is the number of types. The total cost for the regulator to compensate the individuals left aside is bounded above by $|\mathcal{X}| \bar{\Phi}$.

Proof. See appendix.

\section{Matching in Large Numbers}

We now consider the case of a "large" game, in which there are "many" agents of each type. Intuitively, even though an odd number of agents in any type may result in non existence of a stable roommate matching, the resulting game becomes "close" to one in which a stable matching exists. We now flesh out this intuition by providing a formal analysis.

We start with a formal definition of a large game. For that purpose, we consider a sequence of games with the same number of types and the same surplus matrix, but with increasing populations in each type. If $n_{x}^{k}$ denotes the population of type $x$ in game $k$ and $N^{k}=\sum_{x} n_{x}^{k}$ is the total population of that game, then we consider situations in which, when $k \rightarrow \infty$ :

$$
N^{k} \rightarrow \infty \text { and } n_{x}^{k} / N^{k} \longrightarrow f_{x}
$$

where $f_{x}$ are constant numbers.

As the population gets larger, aggregate surplus increases proportionally; it is therefore natural to consider the average surplus, computed by dividing aggregate surplus by the size of the population. We also extend the definition of $\mathcal{W}_{\mathcal{B}}$ in program (7) to non-integers in the obvious way so as to define the limit average bipartite problem $\mathcal{W}_{\mathcal{B}}(f, f, \Phi / 2)$. Note that the linearity of the program implies

$$
\mathcal{W}_{\mathcal{B}}(c n, c m, \Phi / 2)=c \mathcal{W}_{\mathcal{B}}(n, m, \Phi / 2)
$$

for any $c>0$. 
Proposition 5 In the large population limit, under Assumption 1, the average surplus in the roommate matching problem converges to the limit average surplus in the related bipartite matching problem. That is,

$$
\lim _{k \rightarrow \infty} \frac{\mathcal{W}_{\mathcal{P}}\left(n^{k}, \Phi\right)}{N^{k}}=\lim _{N^{k} \rightarrow \infty} \frac{\mathcal{W}_{\mathcal{B}}\left(n^{k}, n^{k}, \Phi / 2\right)}{N^{k}}=\mathcal{W}_{\mathcal{B}}(f, f, \Phi / 2)
$$

Proof. See appendix.

Our approximation results crucially rely on the number of types becoming small relative to the total number of individuals. By definition, two individuals of the same type are indistinguishable in our formulation, both in their preferences and in the way potential partners evaluate them. This may seem rather strong; however, a closer look at the proof of Theorem 5 shows that our bound can easily be refined. In particular, we conjecture that with a continuum of types, Theorem 5 would hold exactly.

A related effect of the number of individuals becoming much larger than the number of types is that the costs of the policy to restore stability in Theorem 4 become negligible:

Proposition 6 In the large population limit and under Assumption 1,

(i) one may remove a subpopulation of asymptotically negligible size in order to restore the existence of stable matchings.

(ii) the average cost per individual of restoring the existence of stable matchings tends to zero.

Proof. See appendix.

In particular, in the case of a continuum of individuals (that is, when there is a finite number of types and an infinite number of individuals of each type), we recover the results of Azevedo et al., 2013 (hereafter, AWW). To make the connection with this paper, the partner types in our setting translates into goods in AWW's. The social welfare in our setting translates into the utility $u$ of a single consumer in AWW. $u$ is such that $u(C)=$ $\Phi(\{x, y\})$ for $C=\{x, y\}, u(\{x\})=0$, and $u=-\infty$ elsewhere (or very negative). Then it can be shown without difficulty that the existence of a TU stable matching in our setting is equivalent to the existence of a Walrasian equilibrium in the AWW setting. Thus existence and TU stability in the case of a continuum of individuals follows from Theorem and Proposition in AWW. 


\section{The Nonexchangeable Roommate Problem ${ }^{7}$}

We now investigate what happens when the surplus $\Phi_{x y}$ is not necessarily symmetric. This will arise when the roles played by the partners are not exchangeable. For instance, a pilot and a copilot on a commercial airplane have dissymmetric roles, but may be both chosen from the same population. Hence, in this section, we shall assume away Assumption 1, and we refer to the "nonexchangeable roommate problem"; it contains the exchangeable problem as a special case.

As it turns out, this can be very easily recast in the terms of an equivalent symmetric roommate problem. Indeed if $\Phi_{x y}>\Phi_{y x}$, then any match of an (ordered) 2-uple $(y, x)$ will be dominated by a matching of a $(x, y) 2$ uple, and the partners may switch the roles they play and generate more surplus. Therefore, in any optimal (or stable) solution there cannot be such a $(y, x)$ 2-uple. As a consequence, the nonexchangeable roommate problem is equivalent to an exchangeable problem where the surplus function is equal to the maximum joint surplus $x$ and $y$ may generate together, that is

$$
\Phi_{x y}^{\prime}=\max \left(\Phi_{x y}, \Phi_{y x}\right)
$$

and since this is symmetric our previous results apply almost directly. Denoting $\pi_{x y}$ the number of $(x, y)$ pairs (in that order), one has

$$
\begin{aligned}
& \mu_{x y}=\pi_{x y}+\pi_{y x}, x \neq y \\
& \mu_{x x}=\pi_{x x}
\end{aligned}
$$

and obviously, $\pi_{x y}$ need not equal $\pi_{y x}$. The population count equation is

$$
n_{x}=\sum_{y \in \mathcal{X}}\left(\pi_{x y}+\pi_{y x}\right), \forall x \in \mathcal{X}
$$

and the social surplus from a matching $\pi$ is

$$
\sum_{x, y \in \mathcal{X}} \pi_{x y} \Phi_{x y}
$$

so that the optimal surplus in the nonexchangeable problem is

$$
\begin{aligned}
\mathcal{W}_{\mathcal{P}}^{\prime}(n, \Phi)= & \max \sum_{x, y \in \mathcal{X}} \pi_{x y} \Phi_{x y} \\
& \text { s.t. } n_{x}=\sum_{y \in \mathcal{X}}\left(\pi_{x y}+\pi_{y x}\right), \forall x \in \mathcal{X} .
\end{aligned}
$$

\footnotetext{
${ }^{7}$ We are grateful to Arnaud Dupuy for correcting a mistake in a preliminary version of the paper.
} 
The following result extends our previous analysis to the nonexchangeable setting:

Theorem 7 The nonexchangeable roommate matching problem is solved by considering the surplus function

$$
\Phi_{x y}^{\prime}=\max \left(\Phi_{x y}, \Phi_{y x}\right)
$$

which satisfies Assumption 1. Call optimized symmetric problem the problem with surplus $\Phi_{x y}^{\prime}$ and population count $n_{x}$. Then:

(i) the optimal surplus in the nonexchangeable roommate problem coincides with the optimal surplus in the corresponding optimized symmetric problem, namely

$$
\mathcal{W}_{\mathcal{P}}^{\prime}(n, \Phi)=\mathcal{W}_{\mathcal{P}}\left(n, \Phi^{\prime}\right)
$$

(ii) the nonexchangeable roommate problem has a stable matching if and only if the optimized symmetric problem has a stable matching.

Given Theorem 7, all results in Sections 3 and 4 hold in the general (nonexchangeable) case. In particular:

- Theorem 1 extends to the general case: the social surplus in the roommate problem with asymmetric surplus $\Phi_{x y}$ is approximated by a bipartite problem with surplus function $\Phi_{x y}^{\prime}=\max \left(\Phi_{x y}, \Phi_{y x}\right) / 2$, or more formally:

$$
\mathcal{W}_{\mathcal{P}}^{\prime}(n, \Phi) \leq \mathcal{W}_{\mathcal{B}}\left(n, n, \Phi^{\prime} / 2\right) \leq \mathcal{W}_{\mathcal{P}}^{\prime}(n, \Phi)+|\mathcal{X}|^{2} \bar{\Phi}
$$

and as an extension of Proposition 2, equality holds in particular when the number of individuals in each types are all even.

- Theorem 3 extends as well: there is a stable matching in the roommate problem with asymmetric surplus $\Phi_{x y}$ if and only if there is equality in the first equality above, that is:

$$
\mathcal{W}_{\mathcal{P}}^{\prime}(n, \Phi)=\mathcal{W}_{\mathcal{B}}\left(n, n, \Phi^{\prime} / 2\right)
$$

- All the asymptotic results in Section 4 hold true: in the asymmetric roommate problem, there is approximate stability and the optimal matching solves a linear programming problem. 


\section{Conclusion}

From a technical perspective, our results are open to various extensions. First, the empirical tools developed in the bipartite setting, especially for the analysis of the marriage markets (see Choo and Siow, 2006, Chiappori et al., 2017, Fox, 2010, Galichon and Salanié, 2016, to cite only a few ${ }^{8}$ ) can be extended to other contexts where the bipartite constraint is relaxed. These include law firms or doctor practices, but also team jobs such as pilot/copilot (and more generally team sports), as well as "tickets" in US presidential elections, marriage markets incorporating single-sex households, and many others.

To be more specific, assume that the joint surplus $\tilde{\Phi}_{i j}$ generated by a match between two individuals $i$ (of type $x$ ) and $j$ (of type $y$ ) is separable in the sense defined by Chiappori et al., 2017:

$$
\tilde{\Phi}_{i j}=\Phi_{x y}+\varepsilon_{y}^{i}+\eta_{x}^{j}
$$

separability assumes that unobserved heterogeneity terms do not interact in the formation of joint surplus.

If the partnership has symmetric roles, then it is easy to see that $\Phi_{x y}$ must be symmetric in $(x, y)$, and that $\eta$ and $\varepsilon$ must be the same family of random variables:

$$
\tilde{\Phi}_{i j}=\Phi_{x y}+\varepsilon_{y}^{i}+\varepsilon_{x}^{j} .
$$

Apart from this specific restriction, if there is a stable matching then the results of Chiappori et al., 2017 apply: there exist $U$ and $V$ such that $U_{x y}+V_{x y} \equiv \Phi_{x y}$ and in equilibrium, if $i$ of type $x$ and $j$ of type $y$ match then $i$ obtains surplus which stems from the maximization of $U_{x z}+\varepsilon_{z}^{i}$ with respect to $z$, including zero for the singlehood option in the maximization. In addition, symmetry implies that $U_{x y}=V_{y x}$. This boils down the matching equilibrium to a series of simple discrete-choice problems. We refer the reader to Galichon and Salanié, 2017 for a short description of separable models, and to Galichon and Salanié, 2016 for a much more complete study of identification and estimation.

Secondly, while our analysis has been conducted in the discrete case, it would be interesting to extend our results to the case where there is an infinite number of agents with a continuum of types. We conjecture that this could be done, at some cost in terms of the mathematics required ${ }^{9}$. Thirdly,

\footnotetext{
${ }^{8}$ Graham, 2011 has a good discussion of this burgeoning literature.

${ }^{9}$ The relevant tools here come from the theory of optimal transportation, see Villani, 2003 and McCann and Guillen, 2013. For the precise connection between matching
} 
we conjecture that the same "cloning" technique could be applied to matches involving more than two partners - the multipartite reference, in that case, being the "matching for teams" context studied by Carlier and Ekeland, 2010. Moreover, it seems natural to apply this technique when utility is not transferable. One may think of assigning arbitrarily genders to both clones of each type, and considering a bipartite stable matching between the two genders. Such a matching will be stable in the roommate matching framework if the bipartite matching of the cloned populations is symmetric. However, such a symmetric stable bipartite matching of the cloned population may not exist. Therefore, the usefulness of cloning to restore stability in the non-transferable utility version of the roommate problem is an open question.

Finally, some roommate problems involve extensions to situations where more than two partners can form a match; but the two-partner case is a good place to start the analysis. Here, we have shown that when the population is large enough with respect to the number of observable types, the structure of the roommate problem is the same as the structure of the bipartite matching problem. Most empirical applications of matching models under TU use a framework as in this paper in order to understand, depending on the context, how the sorting on a given matching market depends on age, education or income, but also height, BMI, marital preferences, etc. ${ }^{10}$. We leave all this for future research.

On a more substantive front, our conclusions are somewhat mixed. While existence issues may be serious in specific contexts, large markets with a discrete distribution of skills (or human capital) tend to be largely immune from these problems. Specifically, and to put things a bit loosely, two factors make partnerships between workers belonging to the same population more likely to be stable: (i) when individuals can be clustered into a small number of basic categories (the latter being defined by either a given level of human capital or a specific combination of skills), and (ii) when different workers belonging to the same category are "close substitutes" to each other - in the sense that substituting one for the other does not change much the joint surplus created in any partnership. For instance, we expect that medical practices formed by a largish number of doctors with similar specialties

models and optimal transportation theory, see Ekeland, 2010, Gretsky et al., 1999 and Chiappori et al., 2010. It is also worth mentioning the recent contribution of Ghoussoub and Moameni, 2013, which uses the same type of mathematical structure for very different purposes.

${ }^{10}$ See for instance Choo and Siow, 2006, Chiappori and Oreffice, 2008 Chiappori et al., 2012 among many others. 
should be rather stable.

However, our results also imply that, in specific cases, stability may be a serious concern. That would be the case when matching involves individuals who do not have close substitutes, for instance because they each have a rare and specific skill (think of a doctor who is the only expert available on one particular disease). In professional partnerships (or in academia!), management skills may also be very unevenly distributed; and our analysis suggests that partnerships that depend on rare leadership skills are more susceptible to break up. ${ }^{11}$ This would also true of firms that rely on a very charismatic individual for inspiration. The early (1969-84) trajectory of Apple under Steve Jobs may be a case in point. Last but not least, sport teams involving a small number of superstars should exhibit stability issues, especially when several stars are associated within the same team. We are not aware of any systematic, empirical analysis of these issues; however, it is fair to say that casual empiricism seems to support these predictions.

When partnerships are least likely to be stable, firm-specific capital is likely to stabilize a partnership. Regulation may also play a useful role. While we do not pursue this here, one can imagine cases when non-compete or "no poaching" clauses that make mobility more costly could actually be welfare-improving, if the courts allow them. Becker, 1991, p. 330 already cited the ability of homosexual unions to "dissolve without judiciary proceedings, alimony, or child support payments" as one reason why they are less stable than heterosexual unions. This is an interesting topic for further research.

\section{References}

Azevedo, E., Weyl, E., \& White, A. (2013). Walrasian equilibrium in large, quasilinear markets. Theoretical Economics, 8, 281-290.

Balinski, M. (1970). On maximum matchings, minimum coverings, and their connections. In H. Kuhn (Ed.), Proc. of the princeton symposium on mathematical programming. Princeton University Press.

Becker, G. (1973). A theory of marriage, part i. Journal of Political Economy, 81, 813-846.

Becker, G. (1974). A theory of marriage, part ii. Journal of Political Economy, 82, S11-S26.

Becker, G. (1991). A treatise on the family. Harvard University Press.

\footnotetext{
${ }^{11}$ Consultancies are an intermediate case: while junior consultants may be relatively interchangeable, leadership matters in finding clients and conserving them.
} 
Carlier, G., \& Ekeland, I. (2010). Matching for teams. Economic Theory, 42, 397-418.

Chiappori, P.-A., McCann, R., \& Nesheim, L. (2010). Hedonic price equilibria, stable matching, and optimal transport: Equivalence, topology, and uniqueness. Economic Theory, 1-49.

Chiappori, P.-A., \& Oreffice, S. (2008). Birth control and female empowerment: An equilibrium analysis. Journal of Political Economy, 116, 113-140.

Chiappori, P.-A., Oreffice, S., \& Quintana-Domeque, C. (2012). Fatter attraction: Anthropometric and socioeconomic characteristics in the marriage market. Journal of Political Economy, 120, 659-695.

Chiappori, P.-A., \& Salanié, B. (2016). The econometrics of matching models. Journal of Economic Literature, 54, 832-861.

Chiappori, P.-A., Salanié, B., \& Weiss, Y. (2017). Partner choice and the marital college premium. American Economic Review, 10\%, 21072169.

Choo, E., \& Siow, A. (2006). Who marries whom and why. Journal of Political Economy, 114, 175-201.

Chung, K.-S. (2000). On the existence of stable roommate matchings. Games and Economic Behavior, 33, 206-230.

Ciscato, E., Galichon, A., \& Goussé, M. (2015). Like attract like: A structural comparison of homogamy across same-sex and different-sex households [mimeo Sciences Po].

Ehrlich, I., \& Kim, J. (2015). Immigration, human capital formation and endogenous economic growth. Journal of Human Capital, 9, 518563.

Ekeland, I. (2010). Notes on optimal transportation. Economic Theory, 42, $437-459$.

Fox, J. (2010). Identification in matching games. Quantitative Economics, 1, 203-254.

Gale, D., \& Shapley, L. (1962). College admissions and the stability of marriage. American Mathematical Monthly, 69, 9-14.

Galichon, A., \& Salanié, B. (2016). Cupid's invisible hand: Social surplus and identification in matching models [mimeo].

Galichon, A., \& Salanié, B. (2017). The econometrics and some properties of separable matching models. American Economic Review Papers and Proceedings, 10\%, 251-255.

Ghoussoub, N., \& Moameni, A. (2013). A self-dual polar factorization for vector fields. Communications on Pure and Applied Mathematics, 65, 905-933. 
Graham, B. (2011). Econometric methods for the analysis of assignment problems in the presence of complementarity and social spillovers. In J. Benhabib, A. Bisin, \& M. Jackson (Eds.), Handbook of social economics. Elsevier.

Gretsky, N., Ostroy, J., \& Zame, W. (1999). Perfect competition in the continuous assignment model. Journal of Economic Theory, 88, 60118.

Gudmunsson, J. (2014). When do stable roommate matchings exist? a review. Review of Economic Design, 18, 151-161.

Gusfield, D., \& Irving, R. (1989). The stable marriage problem: Structure and algorithms. MIT Press.

Irving, R. (1985). An efficient algorithm for the 'stable roommates' problem. Journal of Algorithms, 6, 577-595.

Karlander, J., \& Eriksson, K. (2001). Stable outcomes of the roommate game with transferable utility. International Journal of Game Theory, 29, $555-569$.

Klaus, B., \& Nichifor, A. (2010). Consistency in one-sided assignment problems. Social Choice and Welfare, 35, 415-433.

Klaus, B., \& Klijn, F. (2010). Smith and rawls share a room: Stability and medians. Social Choice and Welfare, 35, 647-667.

McCann, R., \& Guillen, N. (2013). Five lectures on optimal transportation: Geometry, regularity and applications. In G. Dafni (Ed.), Analysis and geometry of metric measure spaces: Lecture notes of the seminaire de mathematiques superieures. American Mathematical Society.

Mortensen, D., \& Pissarides, C. (1994). Job creation and job destruction in the theory of unemployment. Review of Economic Studies, 61, 397415.

Rodrigues-Neto, A. (2007). Representing roommates' preferences with symmetric utilities. Journal of Economic Theory, 135, 545-550.

Shapley, L., \& Shubik, M. (1971). The assignment game i: The core. International Journal of Game Theory, 1, 111-130.

Sotomayor, M. (2005). The roommate problem revisited [mimeo U. São Paulo].

Talman, D., \& Yang, Z. (2011). A model of partnership formation. Journal of Mathematical Economics, 47, 206-212.

Tan, J. (1991). A necessary and sufficient condition for the existence of a complete stable matching. Journal of Algorithms, 12, 154-178.

Villani, C. (2003). Topics in optimal transportation. American Mathematical Society. 


\section{A Appendix: Proofs}

Our proofs use an auxiliary object: the highest possible surplus for a fractional roommate matching, namely

$$
\mathcal{W}_{\mathcal{F}}(n, \Phi)=\max _{\mu \in \mathcal{F}(n)}\left(\sum_{x} \mu_{x x} \Phi_{x x}+\sum_{x \neq y} \mu_{x y} \frac{\Phi_{x y}}{2}\right) .
$$

where $\mathcal{F}(n)$ is the set of fractional (roommate) matchings, which relaxes the integrality constraint on $\mu$ :

$$
\mathcal{F}(n)=\left\{\left(\mu_{x y}\right):\left(\begin{array}{c}
2 \mu_{x x}+\sum_{y \neq x} \mu_{x y} \leq n_{x} \\
\mu_{x y}=\mu_{y x} \\
\mu_{x y} \geq 0
\end{array}\right)\right\} .
$$

The program (11) has no immediate economic interpretation since fractional roommate matchings are infeasible in the real world; and while obviously $\mathcal{W}_{\mathcal{P}}(n, \Phi) \leq \mathcal{W}_{\mathcal{F}}(n, \Phi)$, the inequality in general is strict. We are going to show, however, that the difference between the two programs vanishes when the population becomes large. Moreover, we will establish a link between (11) and the surplus at the optimum of the associated bipartite matching problem.

We start by proving:

\section{Lemma A.1}

$$
\mathcal{W}_{\mathcal{F}}(n, \Phi)=\mathcal{W}_{\mathcal{B}}(n, n, \Phi / 2) .
$$

Moreover, problem (11) has a half-integral solution.

Proof of Lemma A.1. First consider some fractional roommate matching $\mu \in \mathcal{F}(n)$, and define

$$
\begin{aligned}
& \nu_{x y}=\mu_{x y} \text { if } x \neq y \\
& \nu_{x x}=2 \mu_{x x} .
\end{aligned}
$$

As a (possibly fractional) bipartite matching, clearly $\nu \in \mathcal{B}(n, n)$; and

$$
\sum_{x} \mu_{x x} \Phi_{x x}+\sum_{x \neq y} \mu_{x y} \frac{\Phi_{x y}}{2}=\frac{1}{2} \sum_{x, y \in \mathcal{X}} \nu_{x y} \Phi_{x y} .
$$

Now the right-hand side is the aggregate surplus achieved by $\nu$ in the bipartite matching problem with margins $(n, n)$ and surplus function $\Phi / 2$. It follows that

$$
\mathcal{W}_{\mathcal{F}}(n, \Phi) \leq \mathcal{W}_{\mathcal{B}}(n, n, \Phi / 2)
$$


Conversely, let $\left(\nu_{x y}\right)$ maximize aggregate surplus over $\mathcal{B}(n, n)$ with surplus $\Phi / 2$. By symmetry of $\Phi,\left(\nu_{y x}\right)$ also is a maximizer; and since (7) is a linear program, $\nu_{x y}^{\prime}=\frac{\nu_{x y}+\nu_{y x}}{2}$ also maximizes it. Define

$$
\begin{aligned}
& \mu_{x y}^{\prime}=\nu_{x y}^{\prime} \text { if } x \neq y \\
& \mu_{x x}^{\prime}=\frac{\nu_{x x}}{2} .
\end{aligned}
$$

Then

$$
\begin{aligned}
2 \mu_{x x}^{\prime}+\sum_{y \neq x} \mu_{x y}^{\prime} & =\nu_{x x}+\frac{1}{2} \sum_{y \neq x}\left(\nu_{x y}+\nu_{y x}\right) \\
& =\frac{1}{2}\left(\nu_{x x}+\sum_{y \neq x} \nu_{x y}\right) \\
& +\frac{1}{2}\left(\nu_{x x}+\sum_{y \neq x} \nu_{y x}\right) .
\end{aligned}
$$

Now $\nu_{x x}+\sum_{y \neq x} \nu_{x y} \leq n_{x}$ by the scarcity constraint of "men" of type $x$, and $\nu_{x x}+\sum_{y \neq x} \nu_{y x} \leq n_{x}$ by the scarcity constraint of "women" of type $x$. It follows that $\mu^{\prime} \in \mathcal{F}(n)$, and

$$
\sum_{x} \mu_{x x}^{\prime} \Phi_{x x}+\sum_{x \neq y} \mu_{x y}^{\prime} \frac{\Phi_{x y}}{2}=\frac{1}{2} \sum_{x, y \in \mathcal{X}} \nu_{x y} \Phi_{x y}
$$

Therefore the values of the two programs coincide.

Half-integrality follows from the Birkhoff-von Neumann theorem: there always exists an integral solution $\nu$ of the associated bipartite matching problem, and the construction of $\mu^{\prime}$ makes it half-integral ${ }^{12}$.

Given Lemma A.1, we can now prove Theorem 1.

Proof of Theorem 1. The first inequality simply follows from the fact that $\mathcal{P}(n) \subset \mathcal{F}(n)$. Let us now show the second inequality. Lemma A.1 proved that $\mathcal{W}_{\mathcal{F}}(n, \Phi)=\mathcal{W}_{\mathcal{B}}(n, n, \Phi / 2)$. Let $\mu$ achieve the maximum in $\mathcal{W}_{\mathcal{F}}(n, \Phi)$, so that

$$
\mathcal{W}_{\mathcal{F}}(n, \Phi)=\sum_{x} \mu_{x x} \Phi_{x x}+\sum_{x \neq y} \mu_{x y} \frac{\Phi_{x y}}{2} .
$$

\footnotetext{
${ }^{12}$ The half-integrality of the solution of problem (11) also follows from a general theorem of Balinski, 1970; but the proof presented here is self-contained.
} 
Let $\lfloor x\rfloor$ denote the floor rounding of $x$; by definition, $x<\lfloor x\rfloor+1$, so that

$$
\mathcal{W}_{\mathcal{F}}(n, \Phi)<\sum_{x}\left\lfloor\mu_{x x}\right\rfloor \Phi_{x x}+\sum_{x \neq y}\left\lfloor\mu_{x y}\right\rfloor \frac{\Phi_{x y}}{2}+\sum_{x} \Phi_{x x}+\sum_{x \neq y} \frac{\Phi_{x y}}{2} .
$$

The right-hand side can also be rewritten as

$$
\sum_{x, y}\left\lfloor\mu_{x y}\right\rfloor \Phi_{x y}+\sum_{x, y} \Phi_{x y}
$$

But $\lfloor\mu\rfloor$ is in $\mathcal{B}(n, n)$, and is integer by construction; therefore

$$
\sum_{x, y \in \mathcal{X}}\left\lfloor\mu_{x y}\right\rfloor \Phi_{x y} \leq \mathcal{W}_{\mathcal{P}}(n, \Phi)
$$

Finally,

$$
\sum_{x, y \in \mathcal{X}} \Phi_{x y} \leq|\mathcal{X}|^{2} \Phi
$$

so that

$$
\mathcal{W}_{\mathcal{F}}(n, \Phi) \leq \mathcal{W}_{\mathcal{P}}(n, \Phi)+|\mathcal{X}|^{2} \bar{\Phi}
$$

\section{A.1 Proof of Proposition 2}

Proof. Let $n_{x}^{\prime}=\frac{n_{x}}{2}$. By Lemma A.1, problem $\mathcal{W}_{\mathcal{F}}\left(n^{\prime}, \Phi\right)$ has an halfintegral solution $\mu^{\prime}$; therefore problem $\mathcal{W}_{\mathcal{F}}(n, \Phi)$ has an integral solution $2 \mu^{\prime}$, which must also solve (7). It follows that

$$
\mathcal{W}_{\mathcal{P}}(n, \Phi)=\mathcal{W}_{\mathcal{F}}(n, \Phi)
$$

\section{A.2 Proof of Theorem 3}

Proof. By Theorem A.1, Problem (11) coincides with a bipartite matching problem between marginal $\left(n_{x}\right)$ and itself. By well-known results on bipartite matching, there exist vectors $\left(v_{x}\right)$ and $\left(w_{y}\right)$ such that

$$
\begin{gathered}
v_{x} \geq 0, \quad w_{y} \geq 0 \\
v_{x}+w_{y} \geq \quad \Phi_{x y}
\end{gathered}
$$


and the latter inequality is an equality when $\mu_{x y}>0$. Setting

$$
u_{x}=\frac{v_{x}+w_{x}}{2}
$$

the symmetry of $\Phi$ implies

$$
\begin{aligned}
u_{x} & \geq 0 \\
u_{x}+u_{y} & \geq \Phi_{x y}
\end{aligned}
$$

and

$$
\sum_{x \in \mathcal{X}} n_{x} u_{x}=\sum_{x \in \mathcal{X}} \mu_{x x} \Phi_{x x}+\sum_{x \neq y} \mu_{x y} \frac{\Phi_{x y}}{2}
$$

so that the outcome $(\mu, u)$ is stable.

Conversely, assume that $\mu$ is a stable roommate matching. Then by definition, there is a vector $\left(u_{x}\right)$ such that

$$
\begin{aligned}
u_{x} & \geq 0 \\
u_{x}+u_{y} & \geq \Phi_{x y}
\end{aligned}
$$

and

$$
\sum_{x \in \mathcal{X}} n_{x} u_{x}=\sum_{x \in \mathcal{X}} \mu_{x x} \Phi_{x x}+\sum_{x \neq y} \mu_{x y} \frac{\Phi_{x y}}{2} .
$$

Therefore $(u, A=0)$ are Lagrange multipliers for the linear programming problem (11), and $\mu$ is an optimal solution of (11); finally, $\mu$ is integral since it is a feasible roommate matching. QED.

(i), (ii) and (iii) follow, as there exist integral solutions of (11) if and only if

$$
\mathcal{W}_{\mathcal{P}}(n, \Phi)=\mathcal{W}_{\mathcal{F}}(n, \Phi),
$$

and $\mathcal{W}_{\mathcal{F}}(n, \Phi)$ coincides with $\mathcal{W}_{\mathcal{B}}(n, n, \Phi / 2)$ from Lemma A.1.

\section{A.3 Proof of Theorem 4}

Proof. For each type $x$, remove one individual of type $x$ to the population if $n_{x}$ is odd. The resulting subpopulation differs from the previous one by at most $|\mathcal{X}|$ individuals, and there is an even number of individuals of each type; hence by Proposition 3.1 there exists a stable matching.

Each individual so picked can be compensated with his payoff $u_{x}$. Since $u_{x} \leq \bar{\Phi}$, the total cost of compensating at most one individual of each type is bounded from above by $|\mathcal{X}| \bar{\Phi}$. 


\section{A.4 Proof of Proposition 5}

Proof. By Theorem 1, in the large population limit

$$
\lim _{k \rightarrow \infty} \frac{\mathcal{W}_{\mathcal{P}}\left(n^{k}, \Phi\right)}{N^{k}}=\mathcal{W}_{\mathcal{F}}(f, \Phi)
$$

and Lemma A.1 yields the conclusion.

\section{A.5 Proof of Proposition 6}

Proof. (i) The number of individuals to be removed is bounded from above by $|\mathcal{X}|$, hence its frequency tends to zero as $|\mathcal{X}| / N \rightarrow 0$. (ii) follows from the fact that

$$
\frac{\mathcal{W}_{\mathcal{F}}(n, \Phi)-\mathcal{W}_{\mathcal{P}}(n, \Phi)}{N} \rightarrow 0
$$

\section{A.6 Proof of Theorem 7}

Proof. (i) Consider an optimal solution $\mu_{x y}$ to $\mathcal{W}_{\mathcal{P}}\left(n, \Phi^{\prime}\right)$. For any pair $x \neq y$ such that $\Phi_{x y}>\Phi_{y x}$, set $\pi_{x y}=\mu_{x y}$, and $\pi_{x y}=0$ if $\Phi_{x y}<\Phi_{y x}$. If $\Phi_{x y}=\Phi_{y x}$, set $\pi_{x y}$ and $\pi_{y x}$ arbitrarily nonnegative integers such that $\pi_{x y}+\pi_{y x}=\mu_{x y}$; set $\pi_{x x}=\mu_{x x}$. Then $\pi$ is feasible for the optimized symmetric problem, and one has

$$
\sum_{x \in \mathcal{X}} \mu_{x x} \Phi_{x x}^{\prime}+\sum_{x \neq y} \mu_{x y} \frac{\Phi_{x y}^{\prime}}{2}=\sum_{x, y \in \mathcal{X}} \pi_{x y} \Phi_{x y}
$$

so that

$$
\mathcal{W}_{\mathcal{P}}\left(n, \Phi^{\prime}\right) \leq \mathcal{W}_{\mathcal{P}}^{\prime}(n, \Phi)
$$

Conversely, consider $\pi_{x y}$ an optimal solution to $\mathcal{W}_{\mathcal{P}}^{\prime}(n, \Phi)$. First observe that if $\Phi_{x y}<\Phi_{y x}$ then $\pi_{x y}=0$; otherwise subtracting one from $\mu_{x y}$ and adding one to $\pi_{y x}$ would lead to an improving feasible solution, contradicting the optimality of $\pi$. Set

$$
\begin{aligned}
& \mu_{x y}=\pi_{x y}+\pi_{y x}, x \neq y \\
& \mu_{x x}=\pi_{x x}
\end{aligned}
$$

so that

$$
\sum_{x \in \mathcal{X}} \mu_{x x} \Phi_{x x}^{\prime}+\sum_{x \neq y} \mu_{x y} \frac{\Phi_{x y}^{\prime}}{2}=\sum_{x, y \in \mathcal{X}} \pi_{x y} \Phi_{x y}
$$


and hence

$$
\mathcal{W}_{\mathcal{P}}^{\prime}(n, \Phi) \leq \mathcal{W}_{\mathcal{P}}\left(n, \Phi^{\prime}\right)
$$

(ii) Assume there is a stable matching $\pi_{x y}$ in the nonexchangeable roommate problem. Then if there is a matched pair $(x, y)$ in that order, one cannot have $\Phi_{y x}>\Phi_{x y}$; otherwise the coalition $(y, x)$ would be blocking. Hence one can define

$$
\begin{aligned}
& \mu_{x y}=\pi_{x y}+\pi_{y x}, x \neq y \\
& \mu_{x x}=\pi_{x x}
\end{aligned}
$$

and the matching $\mu$ is stable in the optimized symmetric problem. Conversely, assume that the matching $\mu$ is stable in the optimized symmetric problem. Then it is not hard to see that, defining $\pi$ from $\mu$ as in the first part of (i) above, the matching $\pi$ is stable in the nonexchangeable roommate problem. 